\title{
Comparing Algebraic and Numerical Solutions of Classical Diffusion Process Equations in Computational Financial Mathematics
}

\author{
ANDREAS RUFFING ${ }^{\mathrm{a}, \mathrm{b}, *}$, PATRICK WINDPASSINGER $^{\mathrm{b}}$ and STEFAN PANIG ${ }^{\mathrm{b}}$ \\ ${ }^{a}$ Center for Applied Mathematics and Theoretical Physics, University of Maribor, Krekova Ulica 2, SLO-2000 Maribor, \\ Slovenia; ${ }^{\mathrm{b}}$ Zentrum Mathematik, Technische Universität München, Arcisstrasse 21, D-80333 München, Germany
}

(Received 28 August 2000)

\begin{abstract}
We revise the interrelations between the classical Black Scholes equation, the diffusion equation and Burgers equation. Some of the algebraic properties the diffusion equation shows are elaborated and qualitatively presented. The related numerical elementary recipes are briefly elucidated in context of the diffusion equation. The quality of the approximations to the exact solutions is compared throughout the visualizations. The article mainly is based on the pedagogical style of the presentations to the Novacella Easter School 2000 on Financial Mathematics.
\end{abstract}

Keywords: Diffusion processes; Diffusion equations

\section{INTRODUCTION}

The Black Scholes equation has played and is playing a strong role in mathematical modelling of financial markets. It connects the understanding of option prizing with diffusion models and thus yields a fascinating interaction between phenomena in nature with phenomena in society. For a detailed description concerning the Black Scholes equation, see [10]. For general reference, see $[1-4,6-9,11]$. Discretizations of this equation have also been investigated in detail, and in principle it seems that there is nothing really new about this celebrated equation. Its symmetry properties however are still a remarkable mathematical fact, in detail with respect to its transformation properties into the classical heat or diffusion equation.

In this article, we prosecute the strategy of reducing solutions of the Black-Scholes equation to solutions of the classical diffusion equation as described in detail in $[10,11]$. We give some explicit examples in which we compare the quality of exact algebraic solutions to the classical diffusion equation with solutions obtained by the standard numerical recipes as proposed in [10]. As a

*Corresponding author. e-mail: ruffing@appl-math.tu-muenchen.de, http://www-m6.mathematik.tu-muenchen.de/ ruffing/ 
consequence, we obtain the main result that has been elucidated in great detail on the Novacella Easter School 2000: The conventional recipes proposed in $[10,11]$ yield a fine qualitative accuracy. As for the standard error analysis, we recommend the reader to refer to [5].

The Easter School in Novacella, Italy, was an attempt to communicate questions in the area of discrete dynamics in nature and society to students in mathematical finance, mathematics, to students in computer science and students in physics and to encourage them to scientifically interact.

The homepage of the Novacella Easter School 2000 is www-m1.ma.tum.de/bilder/2000/ostersem_ 4_00

The organization of this article shall now be as follows:

In the second chapter we revise the interrelation between Black-Scholes equation, diffusion equation and Burgers equation.

In the third and the fourth chapter, algebraic solutions and numerical approximations are developed. They are compared at the end of the fourth chapter.

The authors would like to mention in particular that the impressive graphic results were also partially made possible by the help of Tobias Lasser who had addressed the topic of solution strategies to ordinary differential equations during the Novacella Easter School.

\section{EXAMPLES OF EQUATION TRANSFORMS}

The connection between the Black Scholes equation and the diffusion equation belongs to the standard procedures being taught in computational finance. For the convenience of the reader, we are going to state it right here at the very beginning of the article.

The Black-Scholes equation is given by

$$
\frac{\partial C}{\partial t}+\frac{1}{2} \sigma^{2} S^{2} \frac{\partial^{2} C}{\partial S^{2}}+r S \frac{\partial C}{\partial S}-r C=0
$$

where $C$ refes to a call, $S$ denoting the underlying asset, $t$ the time. $\sigma$ refers to the volatility of the underlying asset, $r$ means the interest rate.

By setting

$$
S=E \mathrm{e}^{x} \quad t=T-\frac{\tau}{(1 / 2) \sigma^{2}} \quad C=E v(x, \tau)
$$

we remove the dimensions of the original Black-Scholes PDE which now results in the equation

$$
\frac{\partial v}{\partial \tau}=\frac{\partial^{2} v}{\partial x^{2}}+(k-1) \frac{\partial v}{\partial x}-k v \quad k=\frac{2 r}{\sigma^{2}}
$$

Putting now

$$
v=\mathrm{e}^{\alpha x+\beta \tau} u(x, \tau)
$$

and setting

$$
\alpha:=-\frac{1}{2}(k-1) \quad \beta:=-\frac{1}{4}(k+1)^{2}
$$

we end up with the diffusion equation

$$
\frac{\partial u}{\partial \tau}=\frac{\partial^{2} u}{\partial x^{2}}
$$

where $-\infty<x<\infty, \tau>0$.

Let us show that also another partial differential equation can be reduced to the diffusion equation by standard methods:

The partial differential equation

$$
\frac{\partial u}{\partial t}+u \frac{\partial u}{\partial x}=\frac{\partial^{2} u}{\partial x^{2}}
$$

is nonlinear and usually referred to by the name Burgers equation. It is remarkable that one can linearize this equation in the following way. Assume that $v(x, t)$ is a purely positive function for which the expression

$$
\frac{\partial}{\partial x} \ln (v(x, t))
$$


is well defined for any $(x, t) \in \mathbb{R}^{2}$. Defining the function

$$
\begin{aligned}
& u: \mathbb{R}^{2} \rightarrow \mathbb{R}, \\
& (x, t) \mapsto u(x, t):=-2 \frac{\partial}{\partial x} \ln (v(x, t))
\end{aligned}
$$

one obtains first by evaluating (7) that

$$
u(x, t)=-\frac{2}{v} \frac{\partial v}{\partial x}
$$

We now differentiate the function $u$ with respect to $t, x$ and obtain

$$
\begin{aligned}
& \frac{\partial u}{\partial t}=\frac{2}{v^{2}} \frac{\partial v}{\partial t} \frac{\partial v}{\partial x}-\frac{2}{v} \frac{\partial^{2} v}{\partial t \partial x} \\
& \frac{\partial u}{\partial x}=\frac{2}{v^{2}}\left(\frac{\partial v}{\partial x}\right)^{2}-\frac{2}{v} \frac{\partial v}{\partial x}
\end{aligned}
$$

Now, taking the second derivative of $u$ with respect to $x$, we receive

$$
\frac{\partial^{2} u}{\partial x^{2}}=-\frac{4}{v^{3}}\left(\frac{\partial v}{\partial x}\right)^{3}+\frac{6}{v^{2}} \frac{\partial v}{\partial x} \frac{\partial^{2} v}{\partial x^{2}}-\frac{2}{v} \frac{\partial^{3} v}{\partial x^{3}}
$$

Combining these expressions in the sense of (7), one recognizes the following: The function $u$ we have introduced by $v$ yields a solution to Burgers equation if

$$
\frac{\partial v}{\partial x}\left(\frac{\partial v}{\partial t}-\frac{\partial^{2} v}{\partial x^{2}}\right)=v \frac{\partial}{\partial x}\left(\frac{\partial v}{\partial t}-\frac{\partial^{2} v}{\partial x^{2}}\right)
$$

Thus, if the solution $v$ is a positive solution to the heat resp. diffusion Eq. (6) for any $(x, t) \in \mathbb{R}^{2}$, the function $u$, defined by (9) provides a solution to Burgers equation.

We have now got some motivation to focus on comparing solutions to the diffusion equation which, as we have seen, is closely related to the Black-Scholes equation and to Burgers equation.

\section{ALGEBRAIC SOLUTIONS TO THE DIFFUSION EQUATION}

From a classification viewpoint, the diffusion resp. heat equation is a second order linear partial differential equation which is parabolic,

$$
\frac{\partial u}{\partial t}=\frac{\partial^{2} u}{\partial x^{2}}
$$

In a conventional sense, linearity is understood as usual, i.e., along with the solutions $u_{1}(x, t)$ and $u_{2}$ $(x, t)$ for the equation, all linear combinations $c_{1} u_{1}$ $(x, t)+c_{2} u_{2}(x, t)$ solve the equation as well. The solutions $u(x, t)(u: \mathbb{R} \times \mathbb{R} \rightarrow \mathbb{R})$ model the time dependent temperature in a long, fully insulated bar which is assumed to be one-dimensional.

One obtains the most simple solutions by combining polynomial functions in $x$ and $t$ as follows:

$$
\begin{gathered}
u(x, t)=1 \quad(c \in \mathbb{R}) \\
u(x, t)=x \\
u(x, t)=\frac{1}{2} x^{2}+t
\end{gathered}
$$

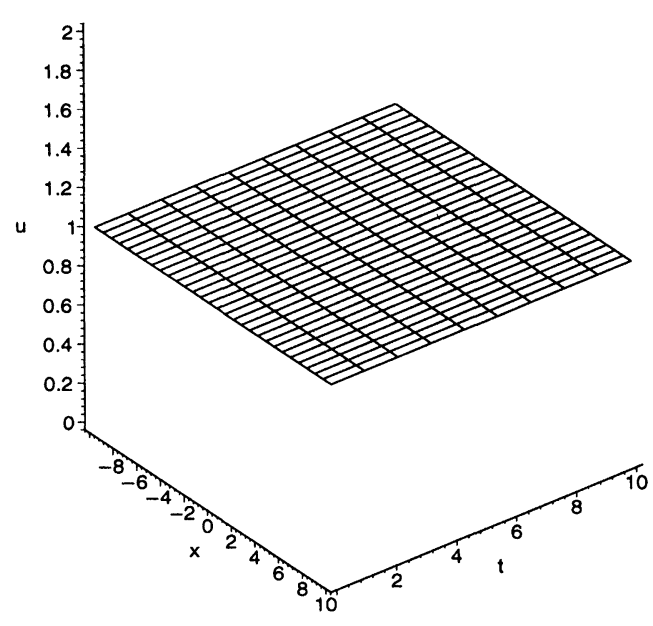

GRAPH $1 u(x, t)=1$. 


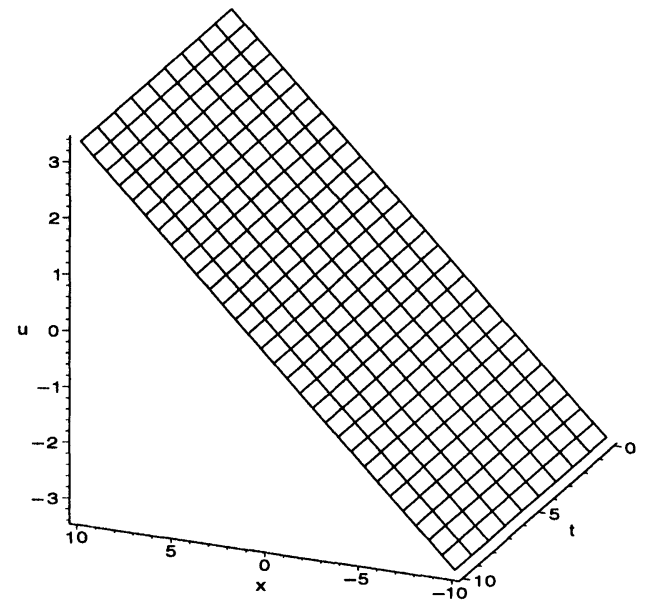

GRAPH $2 u(x, t)=(1 / 3) x$.

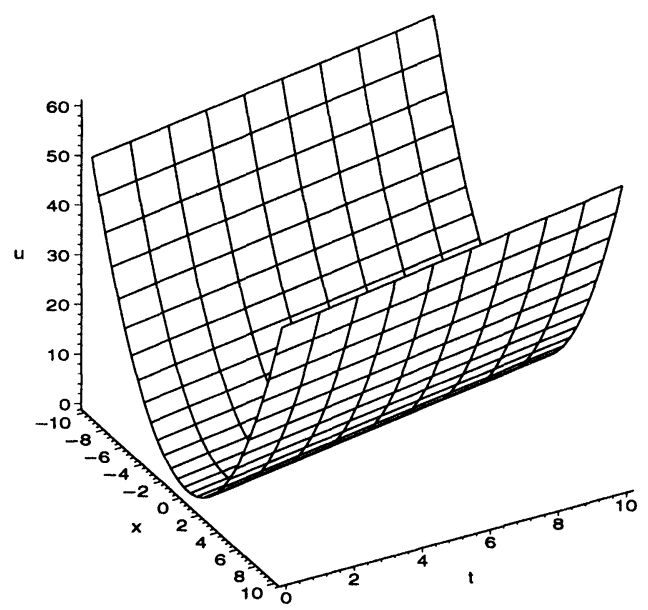

GRAPH $3 u(x, t)=(1 / 2) x^{2}+t$.

Concerning the solution structure, "more diffcult" solutions exist as well, for instance

$$
u(x, t)=c \mathrm{e}^{n x+n^{2} t} \quad(c, n \in \mathbb{R})
$$

as we can easily verify:

$$
\frac{\partial u}{\partial t}=c n^{2} \mathrm{e}^{n x+n^{2} t}=\frac{\partial}{\partial x} c n \mathrm{e}^{n x+n^{2} t}=\frac{\partial^{2} u}{\partial x^{2}}
$$

As $(\partial u / \partial t)=\left(\partial^{2} u / \partial x^{2}\right)$ is a linear differential equation, we obtain other solutions by constructing

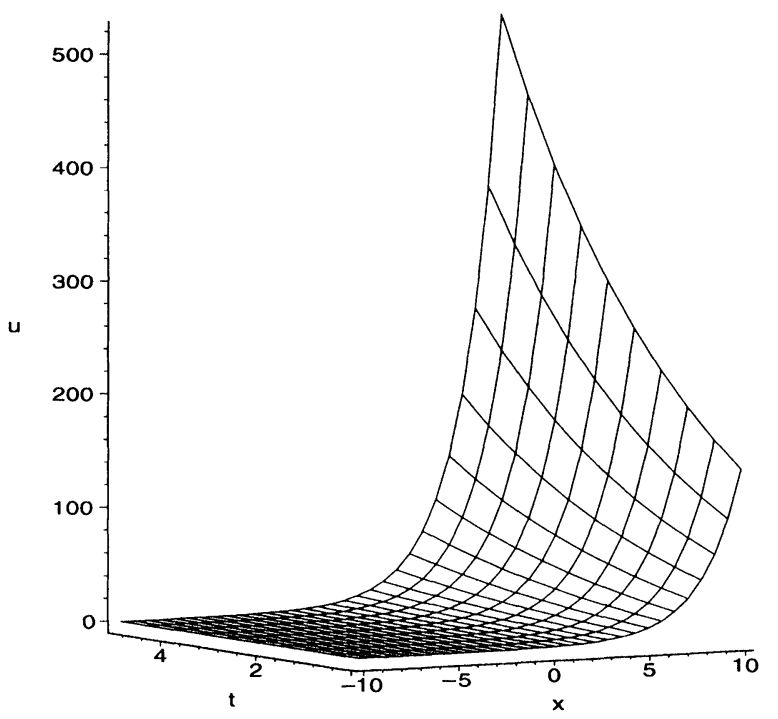

GRAPH $4 u(x, t)=\mathrm{e}^{(1 / 2) x+(1 / 4) t}$.

linear combinations of the particular solutions of type (19):

$$
u(x, t)=\sum_{n=1}^{k} c_{n} \mathrm{e}^{n x+n^{2} t} \quad\left(c_{n} \in \mathbb{R}\right)
$$

This can easily be checked by using induction methods: The case $k=1$ is obviously clear, therefore we address $k-1 \rightarrow k$ :

$$
\begin{aligned}
u_{k}(x, t) & =\sum_{n=1}^{k} c_{n} \mathrm{e}^{n x+n^{2} t}= \\
& =\left(\sum_{n=1}^{k-1} c_{n} \mathrm{e}^{n x+n^{2} t}\right)+c_{k} \mathrm{e}^{k x+k^{2} t}
\end{aligned}
$$

As the partial derivative of a function is linear, $\sum_{n=1}^{k} c_{n} \mathrm{e}^{n x+n^{2} t}$ gives a solution because $\sum_{n=1}^{k-1} c_{n} \mathrm{e}^{n x+n^{2} t}$ satisfies the initial condition. As for $c_{k} \mathrm{e}^{k x+k^{2} t}$ compare (19). Starting the summation at a lower negative index doesn't cause any problems.

We can construct a different solution fulfilling the following boundary conditions:

$$
\begin{aligned}
& u(x, 0)=f(x) \quad 0<x<\pi \\
& u(0, t)=0 \quad t>0 \\
& u(\pi, t)=0 \quad t>0
\end{aligned}
$$




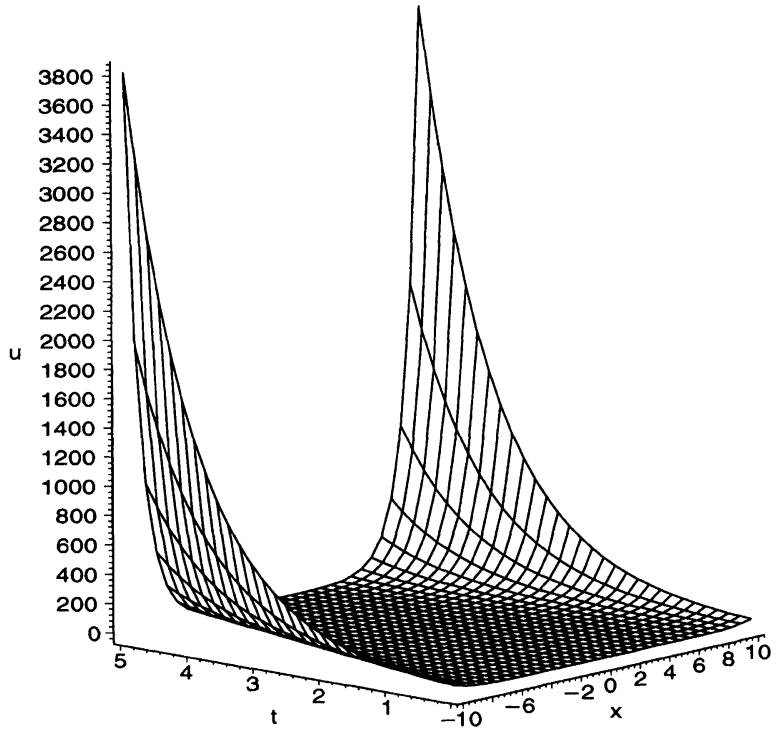

GRAPH $5 u(x, t)=(1 / 1000) \sum_{n=-10}^{10} \mathrm{e}^{(1 / 10) n x+(1 / 100) n^{2} t}$.

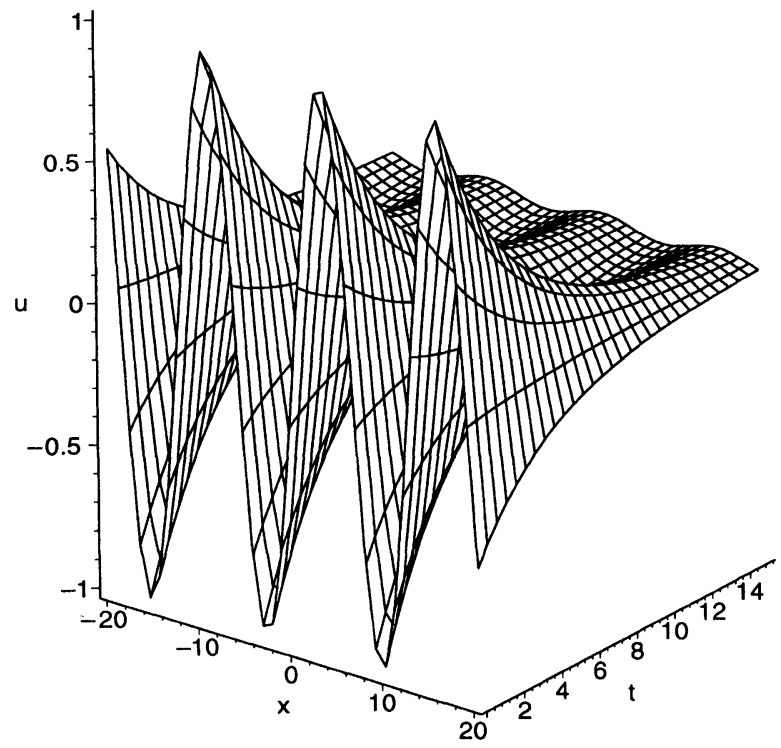

GRAPH $6 u(x, t)=\sin ((1 / 2) x) \mathrm{e}^{-(1 / 4) t}$.

It reads

$$
u(x, t)=c \sin (n x) \mathrm{e}^{-n^{2} t}
$$

as one can explicitly prove:

$$
\begin{aligned}
\frac{\partial u}{\partial t} & =-c n^{2} \sin (n x) \mathrm{e}^{-n^{2} t}= \\
& =\frac{\partial c}{\partial x} c n \cos (n x) \mathrm{e}^{-n^{2} t}=\frac{\partial^{2} u}{\partial x^{2}}
\end{aligned}
$$

Obviously further solutions are obtained by changing sin into cos, sinh or cosh.

Due to the linearity of the heat resp. diffusion equation, the sum again gives a solution:

$$
u(x, t)=\sum_{n=1}^{k} c_{n} \sin (n x) \mathrm{e}^{-n^{2} t} \quad\left(c_{n} \in \mathbb{R}\right)
$$

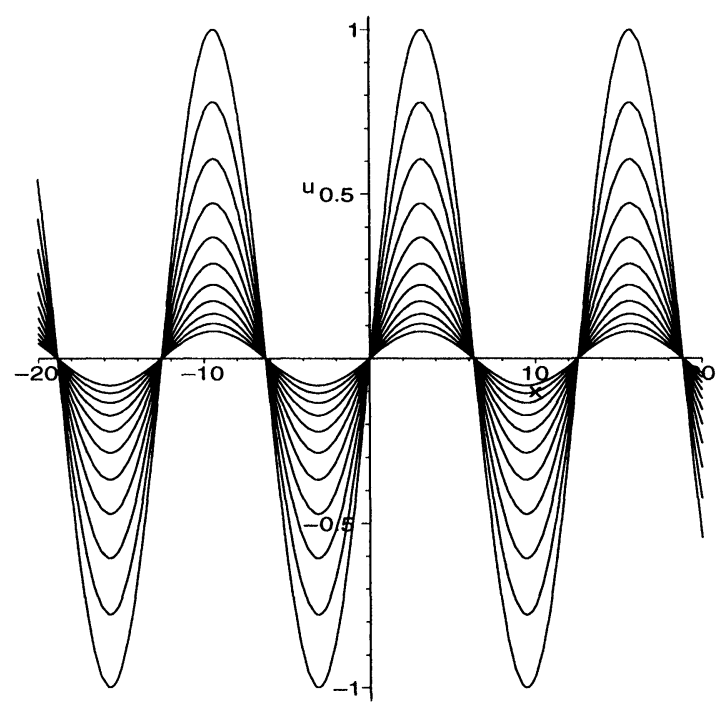

GRAPH $7 u(x, t)=\sin ((1 / 2) x) \mathrm{e}^{-(1 / 4) t}$ with $t=k, k \in[0,10]$.

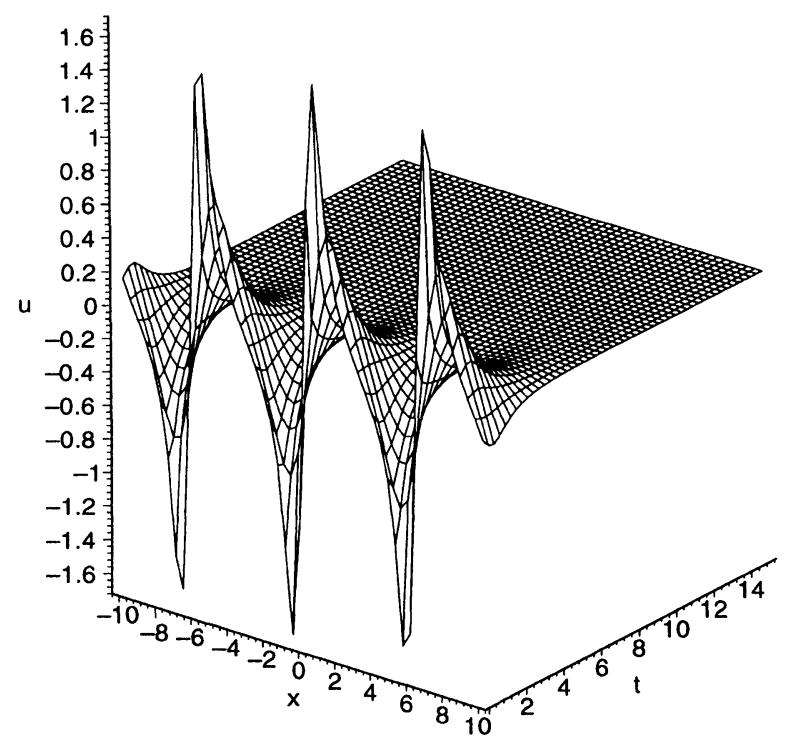

GRAPH $8 \quad u(x, t)=\sum_{n=0}^{100} \sin (n x) \mathrm{e}^{-n^{2} t}$. 


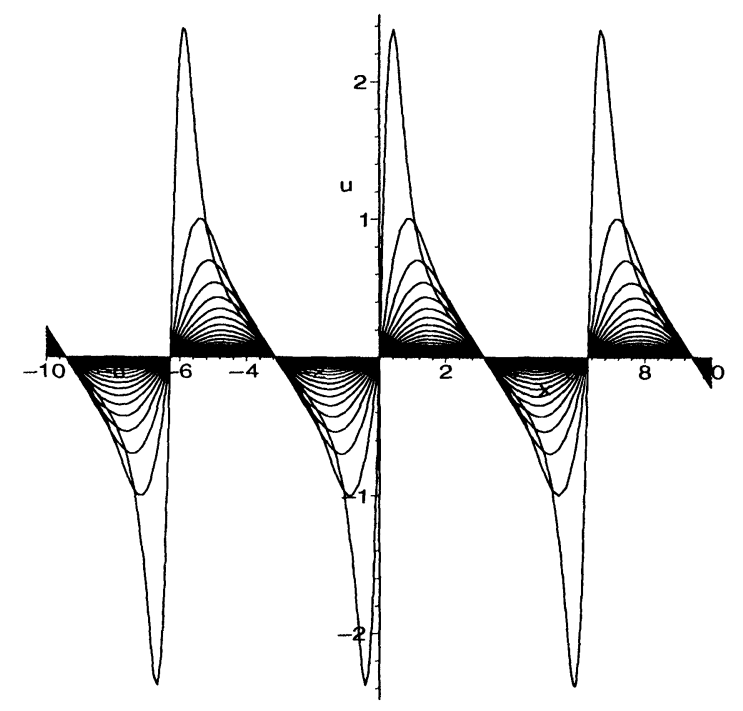

GRAPH $9 u(x, t)=\sum_{n=0}^{100} \sin (n x) \mathrm{e}^{-n^{2} t}$ with $t=0.05+k \cdot 0.2$, $k \in[0,5]$.

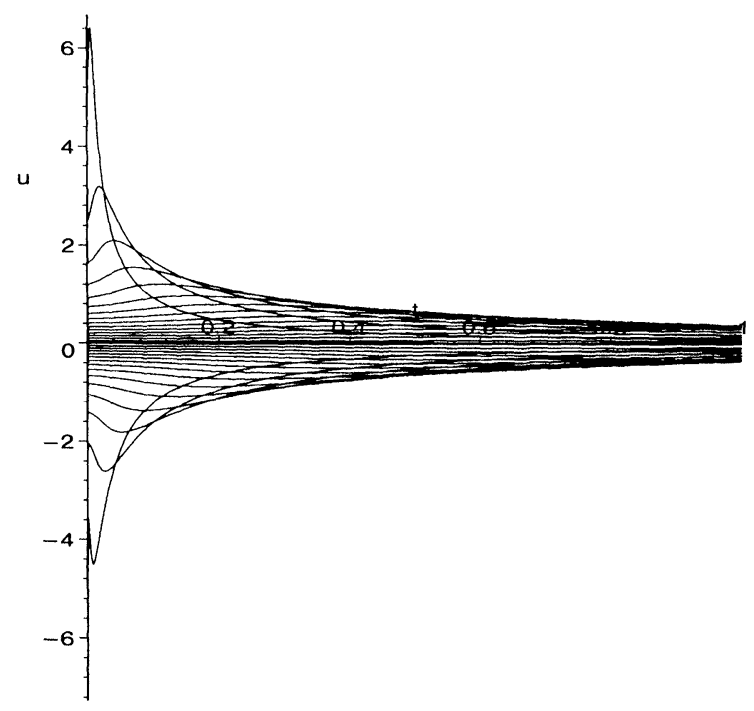

GRAPH $10 u(x, t)=\sum_{n=0}^{100} \sin (n x) \mathrm{e}^{-n^{2} t} \quad$ with $\quad x=0.2 \cdot k$, $k \in[0,30]$.

The proof is similar to the one stated above and one concludes that the sum (22) fulfills the given boundary conditions as well.

From the viewpoint of stochastics, a quite interesting solution is given as follows:

$$
u(x, t)=\frac{c}{\sqrt{t}} \mathrm{e}^{-(1 / 4)\left(x^{2} / t\right)} \quad(t>0, c \in \mathbb{R})
$$

Note that it is very similar to the probability density of the normal distribution $\phi(S)_{\sigma, \mu}=$ $(1 / \sigma \sqrt{2 \pi}) \mathrm{e}^{-(1 / 2)((x-\mu) / \sigma)^{2}}$.

Function (23) yields a solution of the heat equation:

$$
\begin{aligned}
\frac{\partial u}{\partial t}= & -\frac{1}{2} \frac{c}{t^{3 / 2}} \mathrm{e}^{-(1 / 4)\left(x^{2} / t\right)}+\frac{c}{\sqrt{t}} \mathrm{e}^{-(1 / 4)\left(x^{2} / t\right)} \\
& \cdot\left(-\frac{1}{4} \frac{-x^{2}}{t^{2}}\right)= \\
= & \left(\frac{x^{2}}{4 t^{5 / 2}}-\frac{1}{2 t^{3 / 2}}\right) c \mathrm{e}^{-(1 / 4)\left(x^{2} / t\right)} ; \\
\frac{\partial^{2} u}{\partial x^{2}}= & \frac{\partial}{\partial x}\left(\frac{c}{\sqrt{t}} \mathrm{e}^{-(1 / 4)\left(x^{2} / t\right)} \cdot\left(-\frac{x}{2 t}\right)\right)= \\
= & \left(\frac{c}{\sqrt{t}} \mathrm{e}^{-(1 / 4)\left(x^{2} / t\right)} \cdot\left(-\frac{x}{2 t}\right) \cdot\left(-\frac{x}{2 t}\right)\right)+ \\
& +\left(\frac{c}{\sqrt{t}} \mathrm{e}^{-(1 / 4)\left(x^{2} / t\right)} \cdot\left(-\frac{1}{2 t}\right)\right)= \\
= & \left(\frac{x^{2}}{4 t^{5 / 2}}-\frac{1}{2 t^{3 / 2}}\right) c \mathrm{e}^{-(1 / 4)\left(x^{2} / t\right)} ;
\end{aligned}
$$

Looking at the probability density $\phi(s)_{\sigma, \mu}=$ $(1 / \sigma \sqrt{2 \pi}) \mathrm{e}^{-(1 / 2)((x-\mu) / \sigma)^{2}}$ and choosing $\mu=0$, $\phi(s)$ becomes symmetric with respect to the $y$-axis.

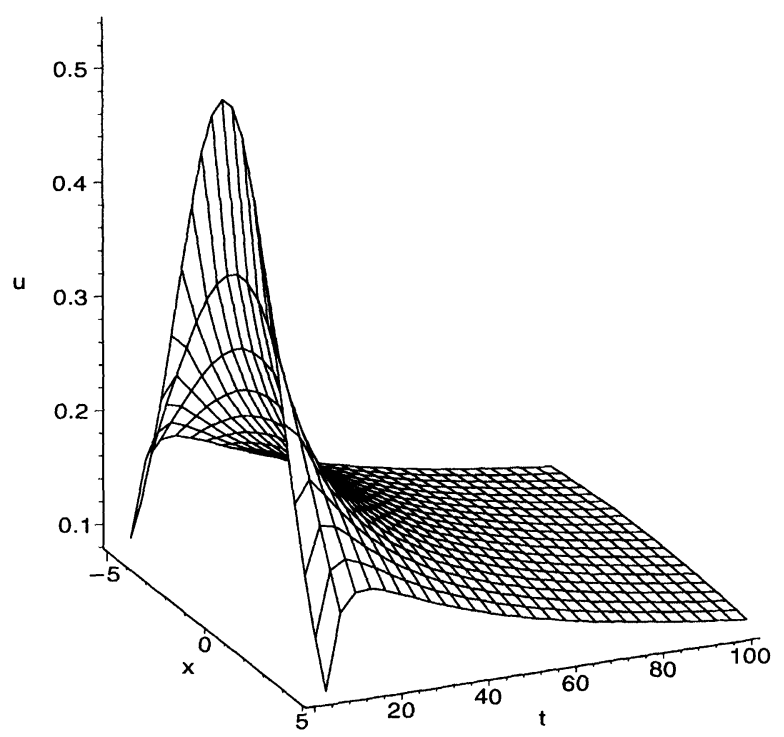

GRAPH $11 u(x, t)=(1 / \sqrt{t}) \mathrm{e}^{-(1 / 4)\left(x^{2} / t\right)}$. 


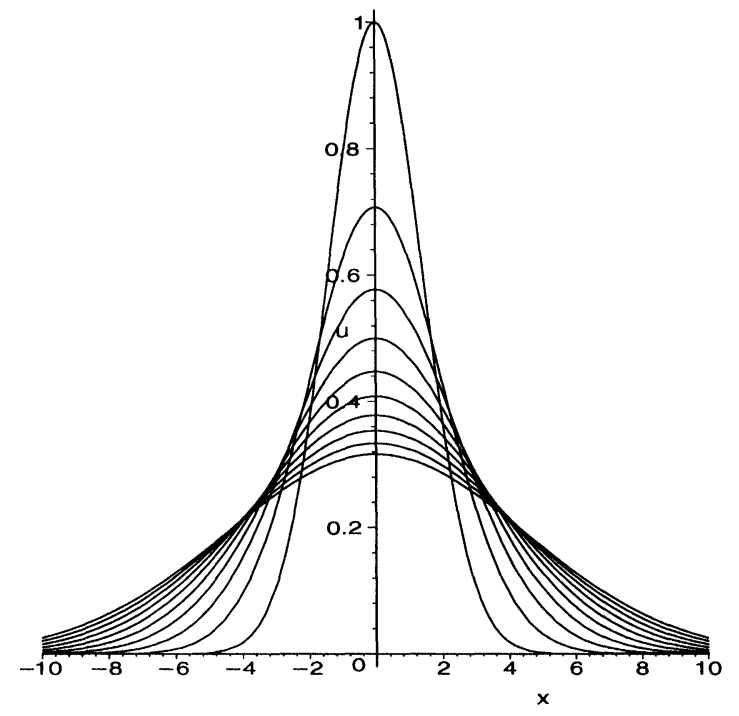

GRAPH $12 u(x, t)=(1 / \sqrt{t}) \mathrm{e}^{-(1 / 4)\left(x^{2} / t\right)}$. with $t=1+k, k \in$ $[0,10]$.

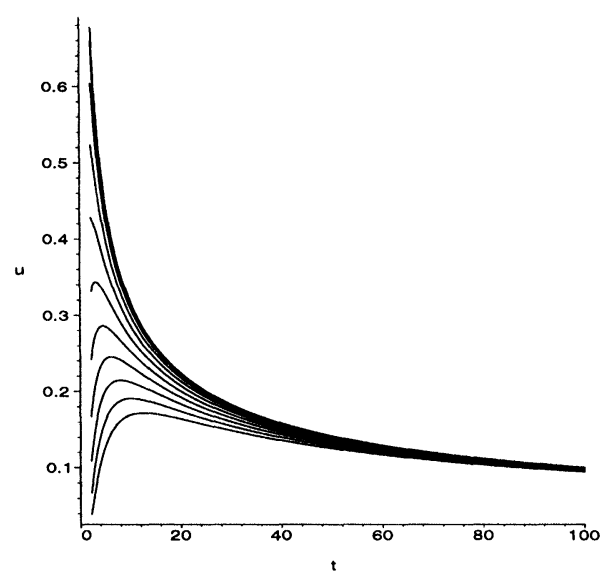

GRAPH $13 u(x, t)=(1 / \sqrt{t}) \mathrm{e}^{-(1 / 4)\left(x^{2} / t\right)} \quad$ with $\quad x=0.5 \cdot k$, $k \in[0,11]$.

If additionally $\sigma^{2}$ is substituted by $2 t$, we exactly get the same result as in (23) with $c=(1 / 2 \sqrt{\pi})$.

The factor $c$ is necessary to ensure that the integral $\Phi=\int_{-\infty}^{+\infty} \phi(s) d s$ equals 1 which reflects the fact that the probability is conserved.

By direct calculation one can verify that the density function with parameter $\mu$ is already a solution of the heat equation when substituting $\sigma^{2}$ by $2 t$ :

$$
\begin{aligned}
\frac{\partial}{\partial t} \phi(x)_{(\sqrt{2 t}, \mu)}= \\
=\frac{\partial}{\partial t} \frac{1}{\sqrt{2 t} \sqrt{2 \pi}} \mathrm{e}^{-(1 / 2)((x-\mu) / \sqrt{2 t})^{2}}= \\
=-\frac{1}{4 \sqrt{\pi}} \frac{1}{t^{3 / 2}} \mathrm{e}^{-(1 / 4)\left((x-\mu)^{2} / t\right)}+ \\
+\left(\frac{1}{2 \sqrt{\pi t}} \mathrm{e}^{-(1 / 4)\left((x-\mu)^{2} / t\right)}\right) \cdot\left(\frac{1}{4} \frac{(x-\mu)^{2}}{t^{2}}\right)= \\
=\left(\frac{1}{8 \sqrt{\pi}} \frac{(x-\mu)^{2}}{t^{5 / 2}}-\frac{1}{4 \sqrt{\pi}} \frac{1}{t^{3 / 2}}\right) . \\
\cdot \mathrm{e}^{-(1 / 4)\left((x-\mu)^{2} / t\right)} ; \\
\frac{\partial^{2}}{\partial x^{2}} \phi(x)(\sqrt{2 t}, \mu) \\
=\frac{\partial}{\partial x} \frac{1}{2 \sqrt{\pi t}} \mathrm{e}^{-(1 / 4)\left((x-\mu)^{2} / t\right) .} \\
\quad \cdot\left(-\frac{1}{4 t} \cdot 2(x-\mu)\right)= \\
=\frac{1}{2 \sqrt{\pi t}} \mathrm{e}^{-(1 / 4)\left((x-\mu)^{2} / t\right)}\left(-\frac{1}{2 t}(x-\mu)\right) . \\
\quad \cdot\left(-\frac{1}{2 t} \cdot(x-\mu)\right)+ \\
\quad+\frac{1}{2 \sqrt{\pi t}} \mathrm{e}^{-(1 / 4)\left((x-\mu)^{2} / t\right)}\left(-\frac{1}{2 t}\right)= \\
=\left(\frac{1}{8 \sqrt{\pi}} \frac{(x-\mu)^{2}}{t^{5 / 2}}-\frac{1}{4 \sqrt{\pi}} \frac{1}{t^{3 / 2}}\right) . \\
\quad \mathrm{e}^{-(1 / 4)\left((x-\mu)^{2} / t\right)} \cdot
\end{aligned}
$$

\section{NUMERICAL APPROXIMATIONS TO THE DIFFUSION EQUATION}

\subsection{Stating the Difference Scheme}

As usual, we replace the partial derivatives by difference quotients where we introduce first order difference quotients and second order difference quotients separately: 


\subsubsection{Derivatives of First Order}

$$
\begin{aligned}
& \frac{\partial u}{\partial \tau}(x, \tau)=\lim _{\delta \tau \rightarrow 0} \frac{u(x, \tau+\delta \tau)-u(x, \tau)}{\delta \tau} \\
& \frac{\partial u}{\partial \tau}(x, \tau)=\lim _{\delta \tau \rightarrow 0} \frac{u(x, \tau)-u(x, \tau-\delta \tau)}{\delta \tau} \\
& \frac{\partial u}{\partial \tau}(x, \tau)=\lim _{\delta \tau \rightarrow 0} \frac{u(x, \tau+\delta \tau)-u(x, \tau-\delta \tau)}{2 \delta \tau} \\
& \text { Contral Difference }
\end{aligned}
$$

The approximation is performed as follows:

$$
\begin{aligned}
\frac{\partial u}{\partial \tau}(x, \tau) & \approx \frac{u(x, \tau+\delta \tau)-u(x, \tau)}{\delta \tau} \approx \\
& \approx \frac{u(x, \tau)-u(x, \tau-\delta \tau)}{\delta \tau} \approx \\
& \approx \frac{u(x, \tau+\delta \tau)-u(x, \tau-\delta \tau)}{2 \delta \tau}
\end{aligned}
$$

$\delta \tau$ is assumed to be sufficiently small - The evaluation for $(\partial u / \partial x)(x, \tau)$ goes in an analogous way.

\subsubsection{Derivatives of Second Order}

Reducing the second order derivatives to the given derivatives of first order yields

$$
\frac{\partial^{2} u}{\partial x^{2}}(x, \tau)=\lim _{\delta x \rightarrow 0} \frac{1}{\delta x}\left(\frac{\partial u}{\partial x}(x+\delta x, \tau)-\frac{\partial u}{\partial x}(x, \tau)\right)
$$

Forward Difference

$$
\frac{\partial^{2} u}{\partial x^{2}}(x, \tau)=\lim _{\delta x \rightarrow 0} \frac{1}{\delta x}\left(\frac{\partial u}{\partial x}(x, \tau)-\frac{\partial u}{\partial x}(x-\delta x, \tau)\right)
$$

Backward Difference

$\frac{\partial^{2} u}{\partial x^{2}}(x, \tau)=\lim _{\delta x \rightarrow 0} \frac{1}{\delta x}\left(\frac{\partial u}{\partial x}\left(x+\frac{\delta x}{2}, \tau\right)-\frac{\partial u}{\partial x}\left(x-\frac{\delta x}{2}, \tau\right)\right)$ Central Difference

$\Rightarrow$ There are three given methods for approximating the first derivative.

$\Rightarrow$ In total we obtain 27 possibilities of approximating the second derivatives in terms of finite differences to the $u$-functions

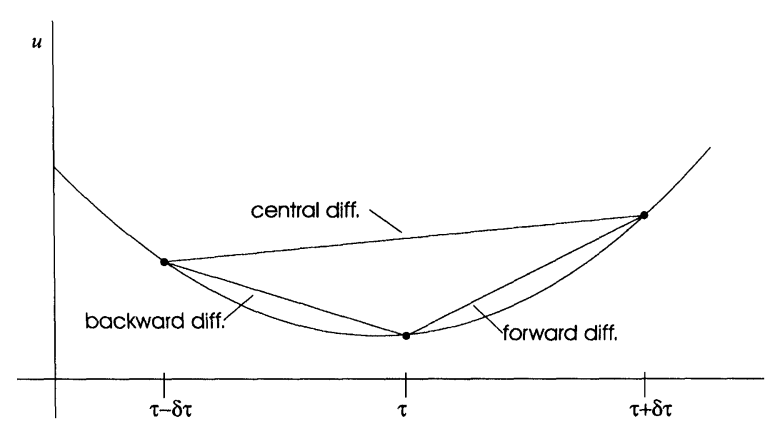

GRAPH 14 Forward Difference, Backward Difference and Central Difference.

In the outlined context, the symmetric central differences are often preferred due to their invariance with respect to the transformations $x \rightarrow-x$ bzw. $\tau \rightarrow-\tau$ :

$$
\begin{aligned}
& \frac{\partial^{2} u}{\partial x^{2}}(x, \tau) \approx \\
& \quad \approx \frac{u(x+\delta x, \tau)-2 u(x, \tau)+u(x-\delta x, \tau)}{(\delta x)^{2}}
\end{aligned}
$$

\subsection{Considering the Errors}

As a consequence of the respective Taylor expansion and the additional assumption that $u(x, \tau)$ allows to calculate its lowest derivatives, we can derive the following equations that give a first insight into the error behavior:

On the one hand, we obtain

$$
u(x, \tau+\delta \tau)=u(x, \tau)+\frac{\partial u}{\partial \tau}(x, \tau)(\delta \tau)+R_{2} \frac{(\delta \tau)^{2}}{2 !}
$$

with

$$
\left|R_{2}\right| \leq \max _{\tau \leq \zeta \leq \tau+\delta \tau}\left|\frac{\partial^{2} u}{\partial \tau^{2}}(x, \zeta)\right|
$$

and on the other hand

$$
\frac{\partial u}{\partial \tau}(x, \tau)=\frac{u(x, \tau+\delta \tau)-u(x, \tau)}{\delta \tau}+O(\delta \tau)
$$




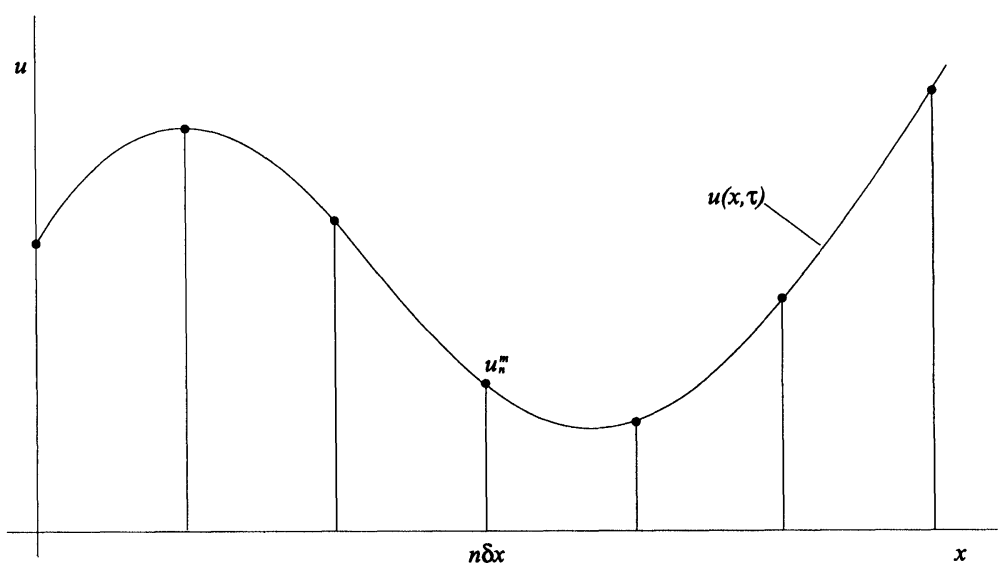

GRAPH 15 Values of $u$ at a fixed time $m \delta \tau$.

respectively

$$
\frac{\partial u}{\partial \tau}(x, \tau)=\frac{u(x, \tau)-u(x, \tau-\delta \tau)}{\delta \tau}+O(\delta \tau)
$$

with

$$
O(\delta \tau)=\frac{1}{2} R_{2} \delta \tau
$$

This means that the error term of the forward resp. backward difference goes to zero in a linear way as $\delta \tau \rightarrow 0$.

Adding the forward and backward difference, we can derive the following approximations from (24):

$$
\frac{\partial u}{\partial x}(x, \tau)=\frac{u(x+\delta x, \tau)-u(x-\delta x, \tau)}{2 \delta x}+O\left((\delta x)^{2}\right)
$$

respectively

$$
\begin{aligned}
& \frac{\partial^{2} u}{\partial x^{2}}(x, \tau)= \\
& \quad=\frac{u(x+\delta x, \tau)-2 u(x, \tau)+u(x-\delta x, \tau)}{(\delta x)^{2}}+ \\
& \quad+O\left((\delta x)^{2}\right)
\end{aligned}
$$

Thus the given central differences yield a quadratic convergence in the stated sense. The stated facts

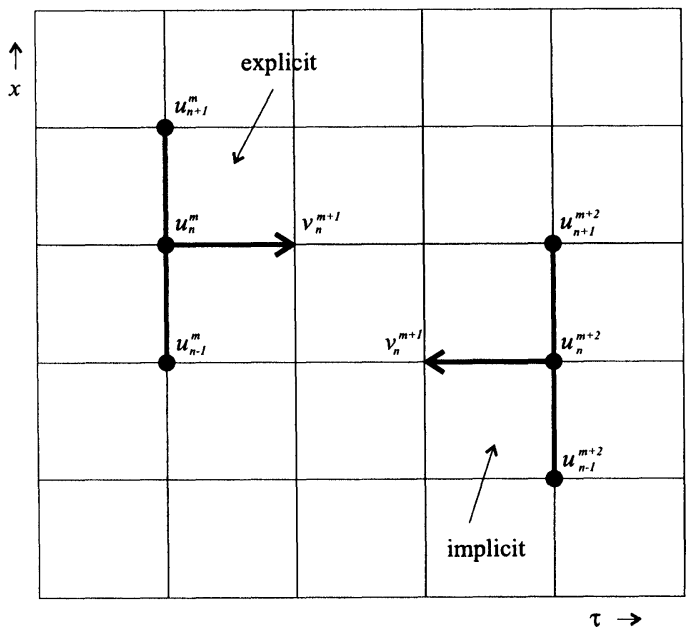

GRAPH 16 Evaluating $v_{n}^{m+1}$ by using explicit resp. implicit methods.

open the possibility of determining exact error bounds.

\subsection{Evaluating the Difference Scheme}

The discretization is performed by choosing an equidistant lattice with mesh lenghts $\delta x$ resp. $\delta \tau$ and lattice coordinates $(n \delta x, m \delta \tau)$ :

$$
u_{n}^{m}=u(n \delta x, m \delta \tau)
$$



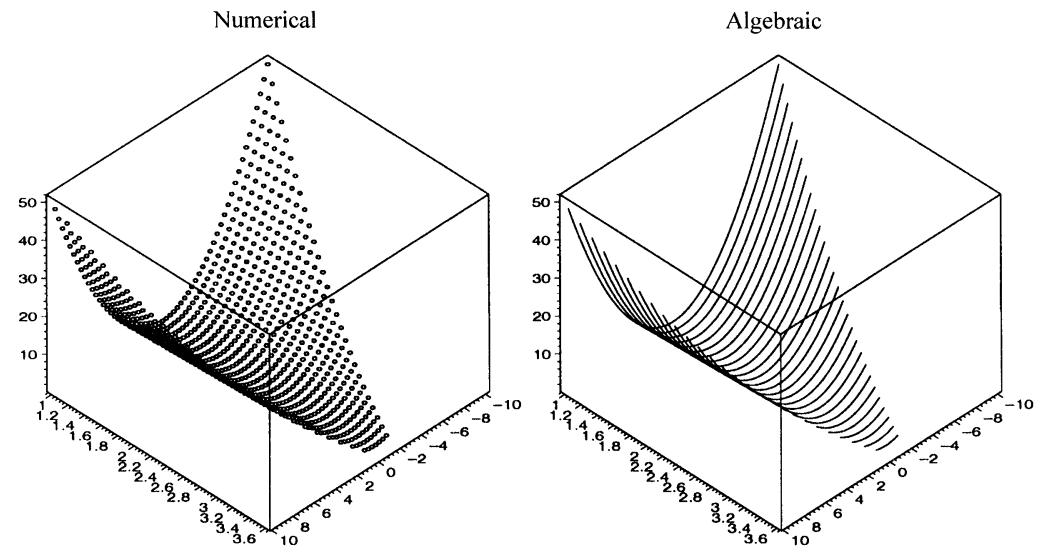

GRAPH $17 u(x, t)=(1 / 2) x^{2}+t$.
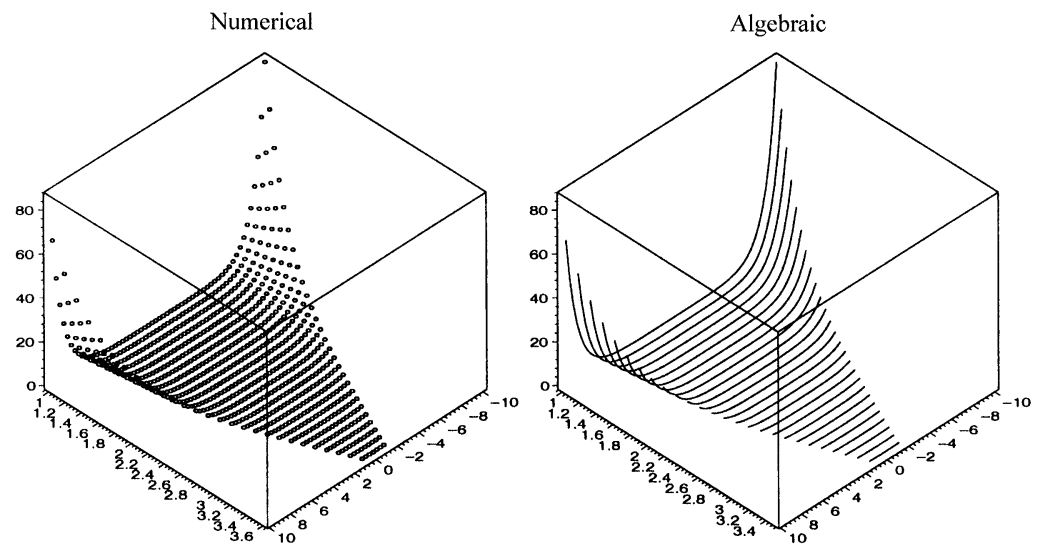

GRAPH $18 u(x, t)=(1 / 1000) \sum_{n=-10}^{10} \mathrm{e}^{(1 / 10) n x+(1 / 100) n^{2} t}$.
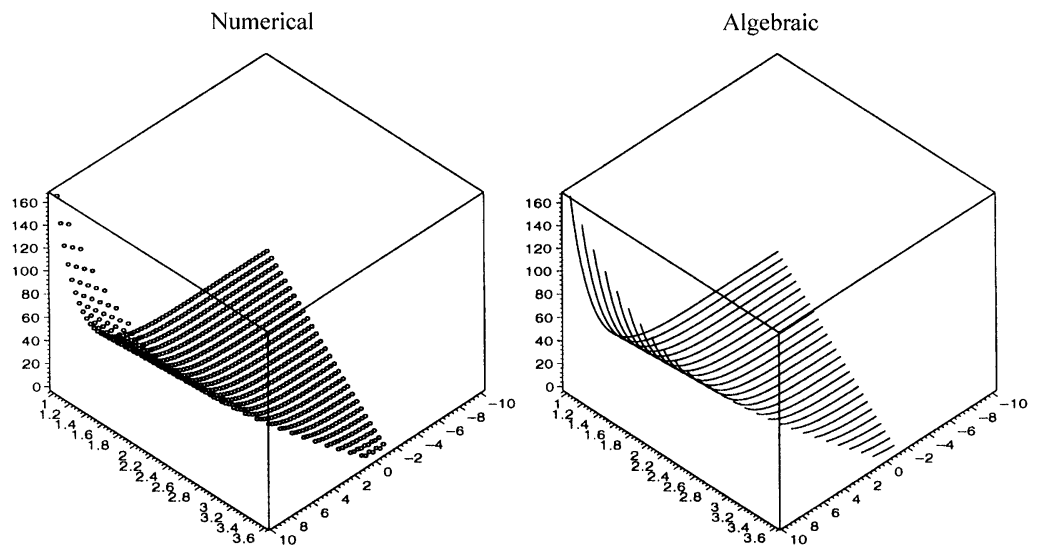

GRAPH $19 u(x, t)=\mathrm{e}^{(1 / 2) x+(1 / 4) t}$. 

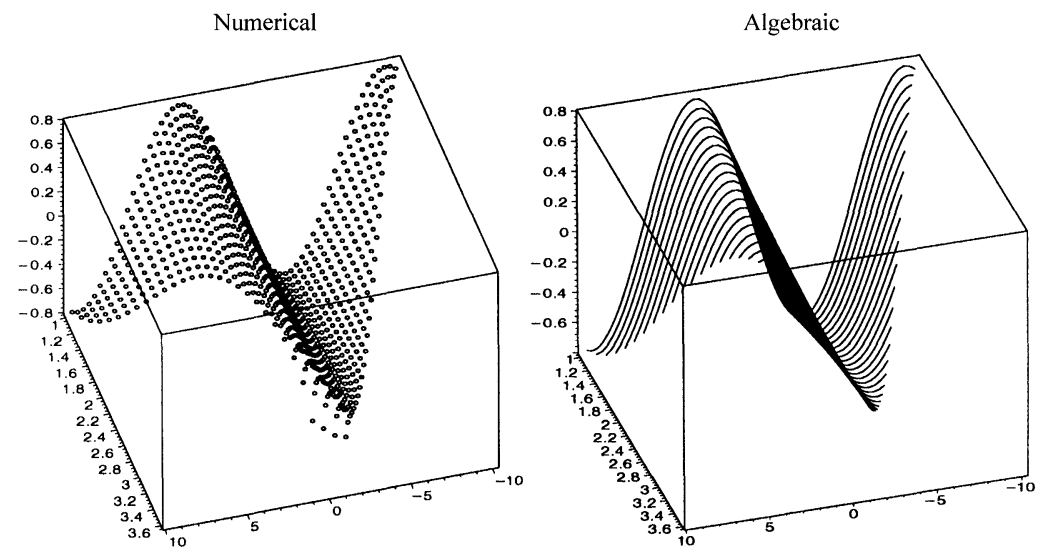

GRAPH $20 u(x, t)=\sin ((1 / 2) x) \mathrm{e}^{-(1 / 4) t}$.
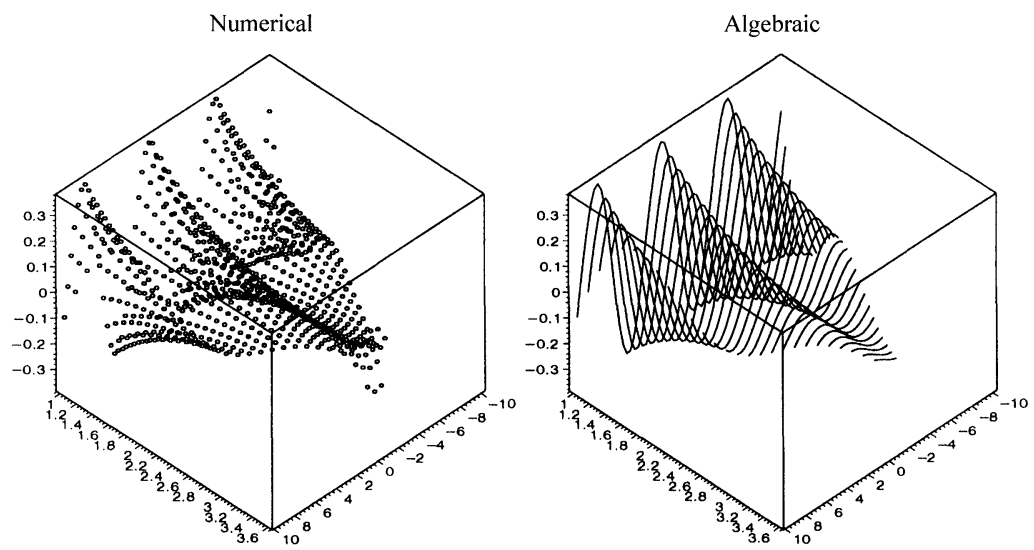

GRAPH $21 \quad u(x, t)=\sum_{n=0}^{100} \sin (n x) \mathrm{e}^{-n^{2} t}$.
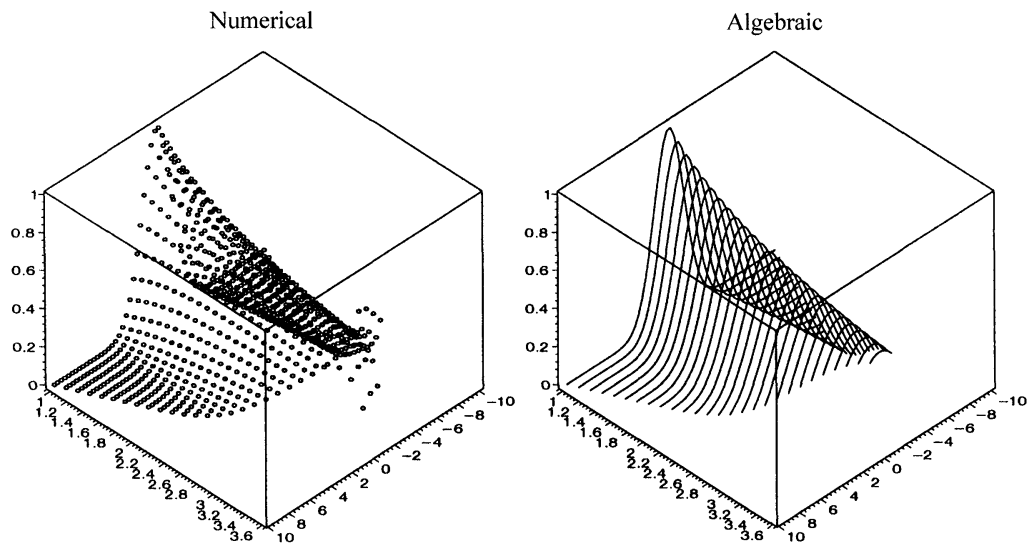

GRAPH $22 u(x, t)=(1 / \sqrt{t}) \mathrm{e}^{-(1 / 4)\left(x^{2} / t\right)}$ 


\subsubsection{The Explicit Approach}

Inserting the forward and symmetric backward central difference into the heat equation now yields

$$
\begin{aligned}
& \frac{u(x, \tau+\delta \tau)-u(x, \tau)}{\delta \tau}+O(\delta \tau)= \\
& =\frac{u(x+\delta x, \tau)-2 u(x, \tau)+u(x-\delta x, \tau)}{(\delta x)^{2}}+ \\
& \quad+O\left((\delta x)^{2}\right)
\end{aligned}
$$

i.e.,

$$
\begin{aligned}
& \frac{u_{n}^{m+1}-u_{n}^{m}}{\delta \tau}+O(\delta \tau)= \\
& =\frac{u_{n+1}^{m}-2 u_{n}^{m}+u_{n-1}^{m}}{(\delta x)^{2}}+O\left((\delta x)^{2}\right)
\end{aligned}
$$

Ignoring the error terms we receive the following approximation formula

$v_{n}^{m+1}=v_{n}^{m}+\alpha\left(v_{n+1}^{m}+v_{n-1}^{m}-2 v_{n}^{m}\right) \quad$ mit $\alpha=\frac{\delta \tau}{(\delta x)^{2}}$

The way it operates is visualized in Graph 16.

\subsubsection{The Implicit Approach}

If we insert the backward difference scheme into Eq. (26) instead of the forward difference scheme, we obtain an implicit difference method.

In the case of the implicit approach, one now makes the following observation: To determine all approximations along the finite lattice, the data along one of its bars are required.

Let us now compare the exact solutions and their approximations.

\section{Acknowledgements}

Andreas Ruffing gratefully appreciates the financial support of the Stifterverband within the award
"Reformfakultät Zentrum Mathematik der Technischen Universität München" to organize the Easter School 2000 in Novacella. He likes to thank Claudia Klüppelberg for arising his interest in several analytical topics of financial mathematics. This work was co-sponsored by the Stadtsparkasse München, the Nova Kreditna Banka Maribor and by Feilmeier and Junker. The financial support by the Ministry of Science and Technology of the Slovenian Government is acknowledged with special thanks. We like to thank in great detail the director of the project "Summerschools" at the Center for Mathematical Sciences at Technical University of Munich, Rupert Lasser. With his help, the Easter School 2000 in Novacella became possible. A lot of thanks are given to Peter Gritzmann for encouraging the organizer of the Easter School at an essential state of the school's origin and for supporting the project as dean of the Center for Mathematical Sciences. The great moral support by Marko Robnik and by Christian Kredler in thinking in an avantgarde way of modern science is acknowledged in particular. The positive attitude of Wolfgang A. Herrmann, President of the Technische Universität München and of Alto Brachner, Director of Natural Sciences at Deutsches Museum, München towards the Summerschool Project of the "Reformfakultät Zentrum Mathematik" is very highly appreciated.

\section{References}

[1] Black, F. and Scholes, M. (1973). The pricing of options and corporate liabilities, J. Pol. Econ., 81, 637-659.

[2] Brennan, M. and Schwartz, E. (1977). Finite-difference methods and jump processes arising in the pricing of contingent claims: a synthesis. J. Finan. Quant. An., 13, $462-474$.

[3] Cox, J. C. and Rubinstein, M. (1985). Option Markets, Prentice Hall.

[4] Friedman, A. (1988). Variational Principles and Free Boundary Problems, Robert E. Krieger Publishing.

[5] Großmann, C. and Roos, H.-G. (1992). Numerik partieller Differentialgleichungen, Teubner.

[6] Harper, J. (1994). Reducing parabolic partial differential equations to canonical form, Europ. J. Appl. Maths., 5, $159-164$. 
[7] Ingersoll, J. E. (1987). Theory of Financial Decision Making, Rowman and Littlefield.

[8] Merton, R. C. (1973). Theory of rational option pricing, Bell J. Econ. Manag. Sci., 4, 141-83.

[9] Stoer, J. and Bulirsch, R. (1993). Introduction to Numerical Analysis, Second edition, Springer.
[10] Wilmott, P., Dewynne, J. and Howison, S. (1995). The Mathematics of Financial Derivatives, A Student Introduction, Cambridge University Press.

[11] Wilmott, P., Dewynne, J. and Howison, S. (1993). Option Pricing: Mathematical Models and Computation, Oxford Financial Press. 


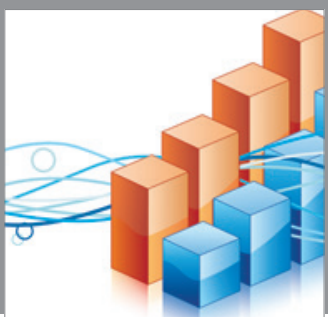

Advances in

Operations Research

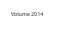

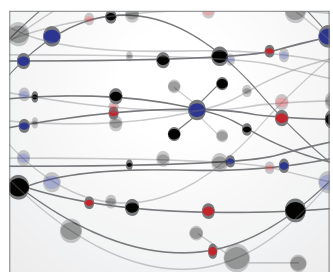

\section{The Scientific} World Journal
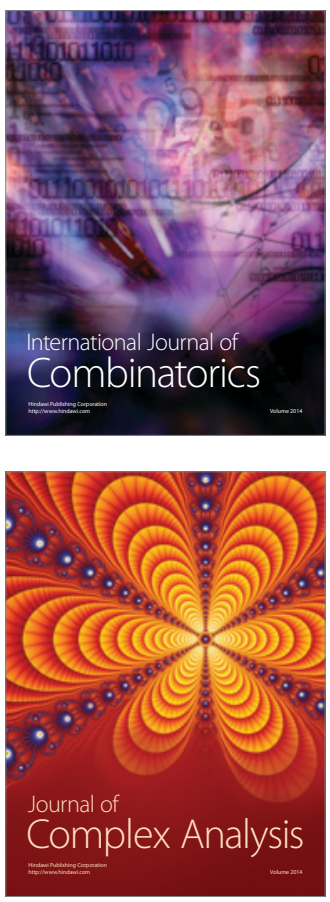

International Journal of

Mathematics and

Mathematical

Sciences
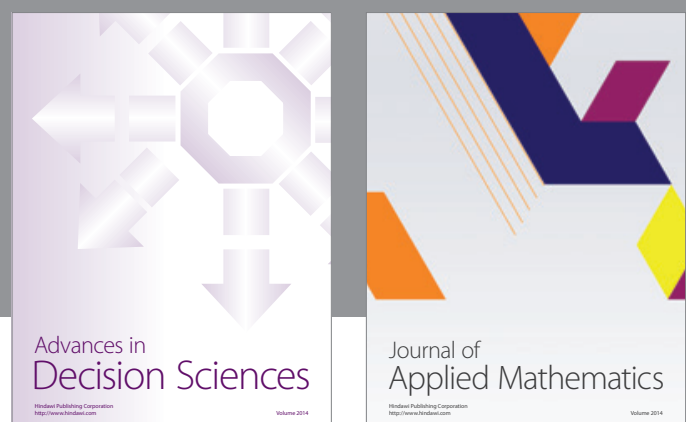

Journal of

Applied Mathematics
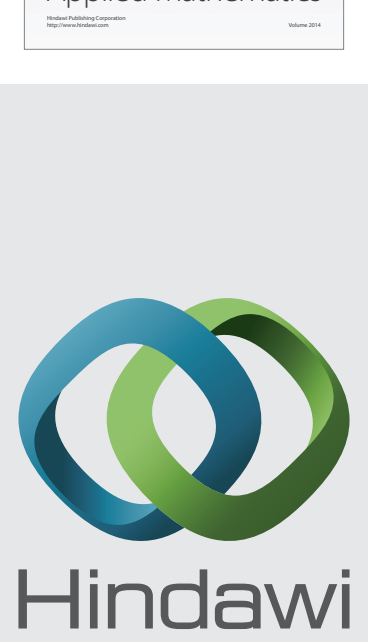

Submit your manuscripts at http://www.hindawi.com
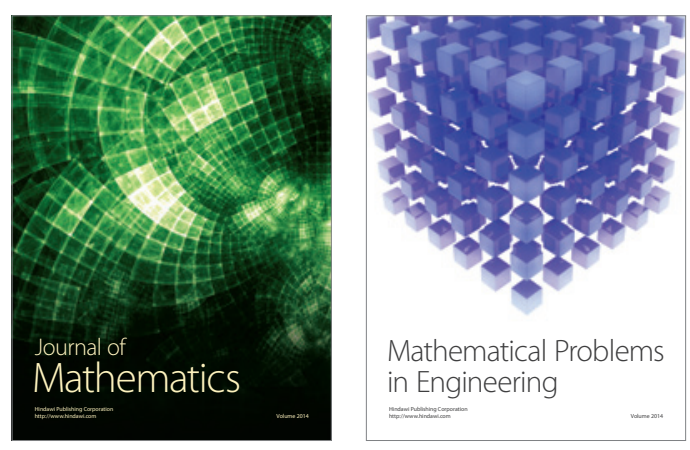

Mathematical Problems in Engineering
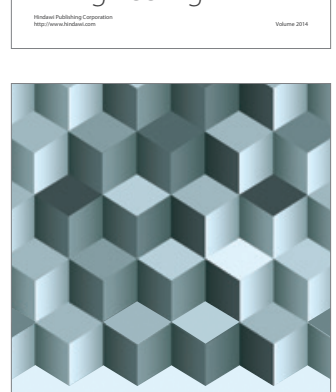

Journal of

Function Spaces
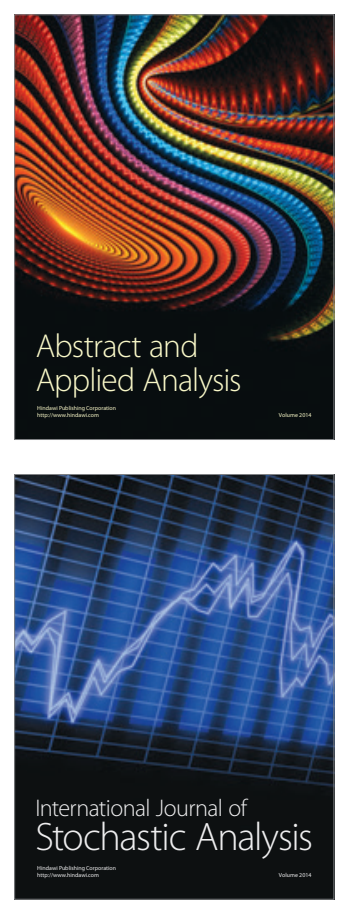

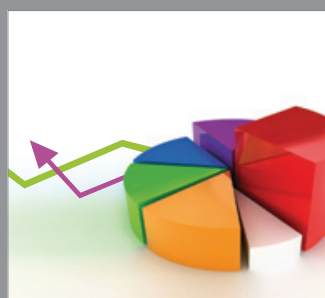

ournal of

Probability and Statistics

Promensencen
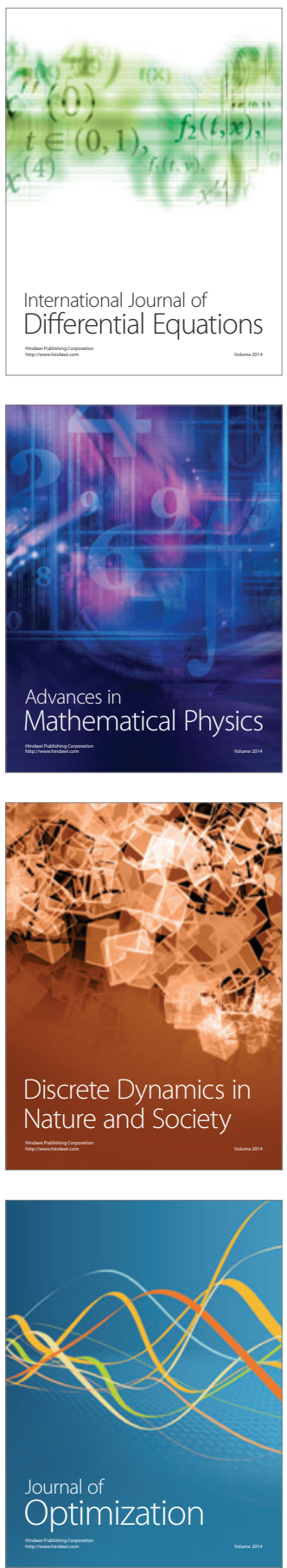